\title{
Hepatic Encephalopathy: Definition, Clinical Grading and Diagnostic Principles
}

\author{
Karin Weissenborn ${ }^{1}$
}

Published online: 31 January 2019

(c) The Author(s) 2019

\begin{abstract}
In general, hepatic encephalopathy (HE) is defined as a brain dysfunction caused by liver insufficiency and/or portal-systemic blood shunting. This article relates to the so-called type C HE: that is, HE in patients with liver cirrhosis. It manifests as a wide spectrum of neurological or psychiatric abnormalities, ranging from subclinical alterations, detectable only by neuropsychological or neurophysiological assessment, to coma. Several scales have been developed for grading the extent of HE. The most often used is the West Haven criteria (WHC), which differentiate between four grades of clinically overt HE. Patients with liver cirrhosis without clinically overt symptoms of HE but neuropsychological or neurophysiological findings indicating brain dysfunction are considered to have minimal hepatic encephalopathy (MHE). For simplification, some experts suggest differentiating between covert HE (MHE plus grade I HE according to WHC) and overt HE (WHC grades II-IV). Diagnosis of both MHE and overt HE is hampered by the fact that none of the symptoms of HE or the findings in the various diagnostic measures applied are specific. Thus, a diagnosis of HE or MHE can only be made after exclusion of other possible causes of brain dysfunction.
\end{abstract}

\section{Definition and Classification}

Hepatic encephalopathy (HE) is a significant complication of severe acute or chronic liver insufficiency that is characterized predominantly by alterations of personality, consciousness, cognition and motor function. Three types of $\mathrm{HE}$ are traditionally differentiated according to the underlying cause: Type A as an essential component of acute liver failure, type $\mathrm{B}$ as a consequence of porto-systemic shunts in the absence of liver dysfunction and type $\mathrm{C}$ in patients with liver cirrhosis and porto-systemic bypass [1]. Current discussion concerns whether HE in patients with acute-on-chronic liver failure should be considered separately (as type D) as it is clinically, pathophysiologically and prognostically distinct from types A-C [2]. This paper is primarily concerned with type C HE.

In the majority of cases, type $\mathrm{C}$ HE occurs episodically in response to well-recognized precipitating factors (Table 1). However, patients may also have a chronic progressive or chronic persistent course of the disorder, especially in the

Karin Weissenborn

weissenborn.karin@mh-hannover.de presence of extensive porto-systemic shunting [3]. These patients may remain in grades I or II HE interspersed with episodes of grade III-IV HE despite therapy. Read et al. [4] described distinct persistent neuropsychiatric syndromes in 21 patients with chronic liver disease and extensive portosystemic shunting: six had a "predominant psychiatric disorder resembling schizophrenia or mania," five had "paraplegia," five had "cerebellar and basal ganglia disorder," three had "paroxysmal disorders of consciousness or muscle spasms" and two had "localized or generalized cortical disease." Data about the prevalence of chronic progressive or persistent HE are sparse. A cross-sectional analysis in 214 patients with liver cirrhosis of different causes revealed cirrhosis-related parkinsonism in $4.2 \%$ of the patients and hepatic myelopathy in $1.9 \%$ [5]. Of note, in contrast to episodic HE, these chronic progressive variants do not respond to the classical ammonia-lowering therapy, indicating a different pathophysiology. However, improvement has been observed after liver transplantation [1].

Each episode of overt HE is associated with an increased risk of further episodes of overt HE and increased 1-year mortality [1]. High-grade HE is also associated with an increased risk of death compared with low-grade HE [2].

1 Clinic for Neurology, Hannover Medical School,

30623 Hannover, Germany 
Table 1 Precipitating factors of hepatic encephalopathy episodes

Infection

Gastrointestinal bleeding

Diuretic overdose

Electrolyte disorder

Constipation

Psychoactive medication

Dehydration

Dietary indiscretion

\subsection{Grading}

The West Haven criteria (WHC) are the most frequently used for grading HE [1]. This grading system differentiates four grades of clinically manifest HE (Table 2). In grade I, patients show a lack of attention and some subtle personality changes that are obvious predominantly to their relatives. In grade II, the most intriguing finding is disorientation for time combined, for example, with inappropriate behavior and lethargy. In grade III, patients are stuporous but respond to stimuli. They are also disoriented for place and situation and may exhibit bizarre behavior. In grade IV, patients are in coma.

When it became obvious that patients without clinical signs of HE may show alterations of brain function in neuropsychological or neurophysiological measures, a fifth grade was added to this system: the so-called subclinical or minimal HE (MHE).

The use of the WHC grading system has been repeatedly challenged, especially its reliability when grading HE I. Though this should be possible with proper clinical assessment, some experts have recommended that MHE and grade I HE be combined and classified as "covert HE" that contrasts to "overt" HE with clinical grades 2-4 [6]. Table 2, which is reprinted with permission from the American Association for the Study of Liver Diseases/ European Association for the Study of the Liver HE guidelines [1], compares these grading systems and provides operative criteria and comments for their use.

Besides alterations of consciousness and cognitive dysfunction, motor symptoms are characteristic features of HE. In particular, motor symptoms include extrapyramidal and cerebellar signs, such as hypomimia, bradykinesia, rigidity, tremor, dysarthria, ataxia and flapping tremor (asterixis), which is probably the most often cited motor symptom of HE. Of note, these symptoms are not strictly tied to a distinct grade of HE. Asterixis, for example, may be present in the absence of alterations of cognition or consciousness. Thus, grading-if necessary-should be done in terms of level of consciousness.

\section{Minimal/Covert Hepatic Encephalopathy: Psychometric Test Procedures}

Cognitive dysfunction frequently precedes further symptoms of HE and may be detectable only using psychometric tests. Affected cognitive domains are attention, visuo-constructional abilities and psychomotor speed and accuracy [6].

Since even these subclinical symptoms are significant in the patient's daily life—as outlined by Montagnese and Bajaj [7] in this Supplement—efforts are ongoing to develop a diagnostic procedure for MHE that is sensitive, specific and simple to apply. Different groups prefer different tests, but there is consensus that the tests applied should assess at least two different cognitive domains [1]. MHE is diagnosed if the subject achieves results worse than the -2 standard deviation (SD) range from ageadjusted norms and-when needed-education-adjusted norms in two different tests, if the tests are not part of a standardized battery. Otherwise, the norms for the battery are applicable. According to the literature, tests used for diagnosing MHE include the Number Connection Tests (NCT) A and B, the Digit Symbol Test, the Block Design Test, the Portosystemic Encephalopathy Syndrome (PSE) Test-providing the Psychometric Hepatic Encephalopathy Score (PHES), the Inhibitory Control Test, the Stroop Test, the Scan Test, the Continuous Reaction Time Test, the Cognitive Drug Research (CDR) test battery or the Repeatable Battery for the Assessment of Neuropsychological Status (RBANS) [1, 8]. Only the PSE syndrome test [6] has been developed especially for the diagnosis of minimal HE; none, including the PSE syndrome test, provide results that are specific for HE. It is of utmost importance that norms achieved from the local population are available for the measure applied in any diagnostic approach.

Since norms for the PHES have been previously elaborated in various countries, including Germany, Spain, Italy, France, the UK, Turkey, Denmark and China, the test is frequently used for diagnosing MHE. It comprises five paper-pencil subtests: the NCT A and B, the Digit Symbol Test, the Serial Dotting Test and the Line Tracing Test. The results for each of these subtests are compared with age-related normative data and converted into scores from +1 to -3 depending on how many SDs a test value in the patient differs from the mean in the normal population. Since time needed to finish the task and number of errors in the Line Tracing Test are considered separately, patients can be allocated between +6 and -18 points. Considering the test results in the normal population as well as those of patients with clinically overt HE (OHE), the cut-off between normal and abnormal results was set 
at -4 points for the German version [6]. Note that various versions of the PHES test are used by different groups, so test results should be compared with caution when it is unclear whether identical versions of the battery have been used [9]. Even more important is to consider that the norms provided or used in Italy or Spain, for example, are based on only five test results: for the Line Tracing Test, the time needed and the number of errors are combined and considered as a single factor, the "error-weighted time" [9]. There is even a difference in calculating this "weighted" score. Here, a cut-off $\leq-4$ is recommended using the respective national norms.

Despite these differences, the PHES test is considered a valuable tool for diagnosing MHE worldwide. The sensitivity of the test for diagnosing HE was $96 \%$ and the specificity (per definition) was $100 \%$ when comparing test results of patients with clinically OHE and healthy controls in the validation study [6]: $22 \%$ of the patients without clinical signs of HE achieved abnormal results in the PHES battery and were classified as having MHE. Campagna et al. [10] recently found abnormal PHES results

Table 2 Grading of HE according to the WHC and the competing ISHEN criteria, which contrast covert and overt hepatic encephalopathy

\begin{tabular}{|c|c|c|c|c|}
\hline $\begin{array}{l}\text { WHC including } \\
\text { MHE }\end{array}$ & ISHEN & Description & Suggested operative criteria & Comment \\
\hline \multicolumn{2}{|c|}{ Unimpaired } & $\begin{array}{l}\text { No encephalopathy at all, no } \\
\text { history of HE }\end{array}$ & Tested and proved to be normal & \\
\hline Minimal & \multirow[t]{2}{*}{ Covert } & $\begin{array}{l}\text { Psychometric or } \\
\text { neuropsychological alterations } \\
\text { of tests exploring } \\
\text { psychomotor speed/executive } \\
\text { functions or } \\
\text { neurophysiological alterations } \\
\text { without clinical evidence of } \\
\text { mental change }\end{array}$ & $\begin{array}{l}\text { Abnormal results of established } \\
\text { psychometric or neuropsychological tests } \\
\text { without clinical manifestations }\end{array}$ & $\begin{array}{l}\text { No universal } \\
\text { criteria for } \\
\text { diagnosis. } \\
\text { Local standards } \\
\text { and } \\
\text { expertise required }\end{array}$ \\
\hline Grade I & & $\begin{array}{l}\text { - Trivial lack of awareness } \\
\text { - Euphoria or anxiety } \\
\text { - Shortened attention span } \\
\text { - Impairment of addition or } \\
\text { subtraction }\end{array}$ & $\begin{array}{l}\text { Despite oriented in time and space (see } \\
\text { below), the patient appears to have some } \\
\text { cognitive/behavioural decay with respect } \\
\text { to his/her standard on clinical } \\
\text { examination, or to the caregivers }\end{array}$ & $\begin{array}{l}\text { Clinical findings } \\
\text { usually not } \\
\text { reproducible }\end{array}$ \\
\hline Grade II & \multirow{3}{*}{ Overt } & $\begin{array}{l}\text { - Lethargy or apathy } \\
\text { - Disorientation for time } \\
\text { - Obvious personality change } \\
\text { - Inappropriate behavior }\end{array}$ & $\begin{array}{l}\text { Disoriented for time (at least three of the } \\
\text { followings are wrong: day of the month, } \\
\text { day of the week, month, season, year) +/- } \\
\text { the other mentioned symptoms }\end{array}$ & $\begin{array}{l}\text { Clinical findings } \\
\text { variable but } \\
\text { reproducible to } \\
\text { some extent }\end{array}$ \\
\hline Grade III & & $\begin{array}{l}\text { - Somnolence to semi-stupor } \\
\text { - Responsive to stimuli } \\
\text { - Confused } \\
\text { - Gross disorientation } \\
\text { - Bizarre behavior }\end{array}$ & $\begin{array}{l}\text { Disoriented also for space ( at least three } \\
\text { of the following wrongly reported } \\
\text { :Country, State -or region-, city, place, +/- } \\
\text { the other mentioned symptoms ) }\end{array}$ & $\begin{array}{l}\text { Clinical findings } \\
\text { reproducible to } \\
\text { some extent }\end{array}$ \\
\hline Grade IV & & Coma & Do not respond even to pain stimuli & $\begin{array}{l}\text { Comatose state } \\
\text { usually } \\
\text { reproducible }\end{array}$ \\
\hline
\end{tabular}

Reproduced from Vilstrup et al. [1] with permission from Wiley \& Sons

$H E$ hepatic encephalopathy, ISHEN International Society for Hepatic Encephalopathy and Nitrogen Metabolism, $M H E$ minimal hepatic encephalopathy, WHC West Haven criteria 
in $42.7 \%$ of 295 patients with liver cirrhosis but no OHE [ $n=50(17 \%)$ had grade $1 \mathrm{HE}, 76(26 \%)$ had MHE].

Currently, the PHES test is widely considered a gold standard that should also be used for the evaluation of new approaches to diagnose MHE or covert HE. This has most recently been done for the Animal Naming Test (ANT) [10], in which subjects are asked to name as many animals as possible within $1 \mathrm{~min}$. After rough correction for age and education effects, the authors defined cut-off values for a (simplified) S-ANT score that, in their opinion, could be used for an estimation of a patients' cognitive function. A score of $\geq 15$ indicates a probability of about $80 \%$ that the subject has no cognitive dysfunction, a score of $<10$ indicates the presence of encephalopathy with a probability of about $80 \%$ [10].

Specialized centers frequently use computerized electroencephalogram (EEG) analysis and/or critical flicker frequency (CFF) in addition to psychometric measures for diagnosing MHE [11, 12]. However, widespread use of the EEG as diagnostic tool is impeded by limited access to this method. For CFF assessment, the portable Hepatonorm ${ }^{\circledR}$ analyzer can be used, for example, without specialist knowledge, and thus this method seems more deployable than the EEG.

A combination of different neuropsychological and neurophysiological diagnostic approaches appears rational, since there is no complete overlap in the results when comparing the various measures available [11-15]. Recently, a combination of the currently most frequently used methods-the PHES, EEG and CFF-has been recommended for screening [12].

\section{Diagnostic Principles}

Diagnosis of HE, clinically overt or not, still requires clinical skills. Neither the clinical symptoms observed in patients with HE nor the neuropsychological or neurophysiological findings characteristic for HE are specific. Thus, a diagnosis of HE can be made only after exclusion of other possible causes of brain dysfunction in every individual patient. Finally, response to treatment might be the best measure to prove the diagnosis.

In addition to the underlying liver disease or complications of liver cirrhosis, concomitant disorders must also be considered. Montagnese et al. [16] reported that 95 of 177 patients with cirrhosis and MHE or OHE had comorbidities that could add to their symptoms besides HE. In case of HE episodes, the most important differential diagnoses are hyponatremia, thiamine deficiency, sepsis and intracranial bleeding [1, 17]. When diagnosing MHE, a patient's individual level of cognitive abilities should also be considered if possible. Patients with greater intellectual abilities (or cognitive reserve) may achieve test results within the (lower) normal range even with increasing metabolic disequilibrium [18]. In the case of chronic progressive HE with parkinsonism, Idiopathic Parkinson's disease and multiple system atrophy must be considered. The patient's medical history, a thorough analysis of the clinical findings and brain imaging, including magnetic resonance imaging (MRI) and single photon emission tomography (SPECT), to assess striatal dopamine transporter and dopamine receptor availability are useful tools in these cases [5]. Clinical findings, clinical course, MRI and, eventually, analysis of cerebrospinal fluid allow for quick differential diagnosis of hepatic myelopathy [19].

\section{Imaging}

So far, brain imaging has provided useful insight into the pathophysiology of HE [20]. Details are also provided in the paper by Butterworth [21] on the pathogenesis of HE in cirrhosis in this Supplement. However, to date, no scientifically proven imaging method exists that could unequivocally diagnose HE. Chen et al. [22] recommend diffusion tensor imaging of the brain and mean diffusivity (MD) and fractional anisotropy (FA) maps as potential biomarkers for MHE. They observed significant differences in MD and FA in distinct white matter areas of the brain in patients with MHE compared with patients with cirrhosis but no HE. Moreover, they identified two spatially distributed white matter regions that were jointly significantly predictive of the group membership. Using MD and FA maps across five different thresholds, they showed that using a threshold of 0.75 SDs below mean FA resulted in a classification accuracy of $92.3 \%$. Although these findings are interesting, FA or MD maps cannot be considered reliable biomarkers of HE. Chen at al. [22] excluded patients with "significant" non-hepatic diseases, and we do not yet know whether and how the frequent concomitant disorders in cirrhotic patients might affect MRI findings. Therefore, we still have no diagnostic imaging tool that might help in diagnosing HE other than by exclusion of other possible causes of brain dysfunction.

\section{Conclusion}

Several proposals have been made to provide a functional definition and grading of $\mathrm{HE}$ for clinical purposes. Although the means provided are helpful, they cannot remediate the fact that HE is a complex disorder that cannot be handled or understood by oversimplification. Irrespective of the method used, certain difficulties in diagnosing HE will remain; in 
any individual case, HE must be differentiated from other possible causes of brain dysfunction. A huge step in advancing the understanding and the diagnosis of HE would be the acknowledgement of general rules for diagnosing brain disorders, such as the application of adequate norms for the assessment of neuropsychological or neurophysiological measures or a deliberate handling of the various diagnostic findings, also for diagnosing HE.

\section{Compliance with Ethical Standards}

Funding This supplement to Drugs has been wholly funded by Merz Pharmaceuticals GmbH, Frankfurt, Germany.

Conflict of Interest KW has received grants and personal fees from Boehringer Ingelheim and Abbvie, and personal fees from Merz Pharmaceuticals $\mathrm{GmbH}$, Novartis, and BMS.

Open Access This article is distributed under the terms of the Creative Commons Attribution-NonCommercial 4.0 International License (http://creativecommons.org/licenses/by-nc/4.0/), which permits any noncommercial use, distribution, and reproduction in any medium, provided you give appropriate credit to the original author(s) and the source, provide a link to the Creative Commons license, and indicate if changes were made.

\section{References}

1. Vilstrup H, Amodio P, Bajaj J, Cordoba J, Ferenci P, Mullen KD, et al. Hepatic encephalopathy in chronic liver disease: 2014 Practice Guideline by the American Association for the Study of Liver Diseases and the European Association for the Study of the Liver. Hepatology. 2014;60:715-35.

2. Romero-Gómez M, Montagnese S, Jalan R. Hepatic encephalopathy in patients with acute decompensation of cirrhosis and acuteon-chronic liver failure. J Hepatol. 2015;62(2):437-47.

3. Riggio O, Efrati C, Catalano C, Pediconi F, Mecarelli O, Accornero N, et al. High prevalence of spontaneous portal-systemic shunts in persistent hepatic encephalopathy: a case-control study. Hepatology. 2005;42(5):1158-65.

4. Read A, Sherlock S, Laidlaw J, Walker JG. The neuro-psychiatric syndromes associated with chronic liver disease and an extensive portal-systemic collateral circulation. Q J Med. 1967;36(114):135-50.

5. Tryc AB, Goldbecker A, Berding G, Rümke S, Afshar K, Shahrezaei GH, et al. Cirrhosis-related Parkinsonism: prevalence, mechanisms and response to treatments. J Hepatol. 2013;58(4):698-705.
6. Weissenborn K, Ennen JC, Schomerus H, Rückert N, Hecker H. Neuropsychological characterization of hepatic encephalopathy. J Hepatol. 2001;34(5):768-73.

7. Montagnese S, Bajaj JS. Impact of hepatic encephalopathy in cirrhosis on quality-of-life issues. Drugs 2018. https://doi. org/10.1007/s40265-018-1019-y.

8. Weissenborn K. Diagnosis of minimal hepatic encephalopathy. J Clin Exp Hepatol. 2015;5(Suppl 1):S54-9.

9. Weissenborn K. PHES: one label, different goods?! J Hepatol. 2008;49(3):308-12.

10. Campagna F, Montagnese S, Ridola L, Senzolo M, Schiff $\mathrm{S}$, De Rui M, et al. The animal naming test: an easy tool for the assessment of hepatic encephalopathy. Hepatology. 2017;66(1):198-208.

11. Morgan MY, Amodio P, Cook NA, Jackson CD, Kircheis G, Lauridsen $\mathrm{MM}$, et al. Qualifying and quantifying minimal hepatic encephalopathy. Metab Brain Dis. 2016;31(6):1217-29.

12. Montagnese S, Balistreri E, Schiff S, De Rui M, Angeli P, Zanus G, et al. Covert hepatic encephalopathy: agreement and predictive validity of different indices. World J Gastroenterol. 2014;20(42):15756-62.

13. Dhiman RK, Kurmi R, Thumburu KK, Venkataramarao SH, Agarwal R, Duseja A, et al. Diagnosis and prognostic significance of minimal hepatic encephalopathy in patients with cirrhosis of liver. Dig Dis Sci. 2010;55(8):2381-90.

14. Romero-Gómez M, Córdoba J, Jover R, del Olmo JA, Ramírez M, Rey R, et al. Value of the critical flicker frequency in patients with minimal hepatic encephalopathy. Hepatology. 2007;45(4):879-85.

15. Goldbecker A, Weissenborn K, Hamidi Shahrezaei G, Afshar K, Rümke S, Barg-Hock H, et al. Comparison of the most favoured methods for the diagnosis of hepatic encephalopathy in liver transplantation candidates. Gut. 2013;62(10):1497-504.

16. Montagnese S, Schiff S, Amodio P. Quick diagnosis of hepatic encephalopathy: fact or fiction? Hepatology. 2015;61(1):405-6.

17. Weissenborn K. Challenges in diagnosing hepatic encephalopathy. Neurochem Res. 2015;40(2):265-73.

18. Montagnese S, De Rui M, Angeli P, Amodio P. Neuropsychiatric performance in patients with cirrhosis: Who is "normal"? J Hepatol. 2017;66(4):825-35.

19. Weissenborn K, Tietge UJ, Bokemeyer M, Mohammadi B, Bode U, Manns MP, et al. Liver transplantation improves hepatic myelopathy: evidence by three cases. Gastroenterology. 2003;124(2):346-51.

20. Zhang XD, Zhang LJ. Multimodal MR imaging in hepatic encephalopathy: state of the art. Metab Brain Dis. 2018;33(3):661-71.

21. Butterworth RF. Hepatic encephalopathy in cirrhosis: pathology and pathophysiology. Drugs 2018. https://doi.org/10.1007/s4026 5-018-1017-0.

22. Chen HJ, Chen R, Yang M, Teng GJ, Herskovits EH. Identification of minimal hepatic encephalopathy in patients with cirrhosis based on white matter imaging and Bayesian data mining. AJNR Am J Neuroradiol. 2015;36(3):481-7. 